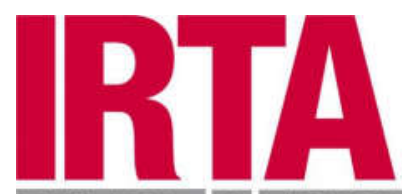

REEGERGA II TIEGNOLOCIA

ACROALIMENTARIIES

This document is a postprint version of an article published in Veterinary Microbiology@ Elsevier after peer review. To access the final edited and published work see https://doi.org/10.1016/j.vetmic.2019.02.034

Document downloaded from:

IRTA Pubpro

Open drital arsive 


\section{Investigation of Haemophilus parasuis from healthy pigs in China}

3 Peng Zhanga, Chaoyang Zhanga, Virginia Aragonb, Xue Zhouc, Ming Zoud,

4 Congming Wua, Zhangqi Shena

5

6 a Beijing Advanced Innovation Center for Food Nutrition and Human Health,

7 College of Veterinary Medicine, China Agricultural University, Beijing, China

8

9 b IRTA, Centre de Recerca en Sanitat Animal (CReSA, IRTA-UAB), Campus de 10 la Universitat Autònoma de Barcelona, Bellaterra, Spain

12 c Chongqing Academy of Animal Science, Chongqing, China

14 d College of Veterinary Medicine, Qingdao Agricultural University, Qingdao,

15 China

\section{Highlights}

- Updated the epidemiologic data of Haemophilus parasuis from healthy pigs in China. 
- Serovars distribution was different from previous reports and showed regional difference.

- Elevated MICs were observed for majority of the tested antimicrobial agents.

Abstract

Haemophilus parasuis is a common colonizer of the upper respiratory tract of swine and frequently causes disease, especially in weaner pigs. To date, limited epidemiological data was available for $H$. parasuis from healthy pigs, which might be carriers of potential pathogenic strains. In this study, from September 2016 to October 2017, we investigated the prevalence and characteristics of $H$. parasuis from healthy pigs in China. Totally, we obtained 244 isolates from 1675 nasal samples from 6 provinces. H. parasuis isolation was more successful in weaner pigs $(22.6 \%, 192 / 849)$, followed by finisher pigs $(9.3 \%$, $43 / 463)$, and sows $(2.5 \%, 9 / 363)$. The most prevalent serovars were $7(20.1 \%$, 49/244), followed by $3(14.8 \%, 36 / 244), 2$ (14.3\%, 35/244), 11 (12.7\%, 31/244), $5 / 12(5.7 \%, 14 / 244)$ and $4(2.5 \%, 6 / 244)$. Bimodal or multimodal distributions of MICs were observed for most of the tested drugs, which suggested the presence of non-wild type populations. It was noted that the MIC90 values of tilmicosin $(64 \mu \mathrm{g} / \mathrm{ml})$ was relatively higher than that reported in previous studies. Our results suggest that: 1) potentially pathogenic serovars of $H$. parasuis are identified in healthy pigs, and 2) elevated MICs and presence of mechanisms of 
45 resistance not yet described for clinically important antimicrobial agents would

46 increase the burden of disease caused by H. parasuis.

47

48

49 Keywords

50

51 Prevalence

52 Healthy pigs

53 Antimicrobial susceptibility

54 Serovars distribution

55 
Haemophilus parasuis is an important swine pathogen that causes serious diseases, such as Glässer's disease, pneumonia and septicemia (Oliveira and Pijoan, 2004). In the United States, $H$. parasuis was the leading bacterial health challenge for nursery pigs (Holtkamp et al., 2007). The Animal and Plant Health Agency (APHA) reports that the annual incidence of $H$. parasuis disease shows a steady increasing trend (except 2012 and 2014) in England, Wales, and Scotland, from nearly $8 \%$ in 2002 to $14 \%$ in 2016 (APHA, 2016). H. parasuis is a common resident organism of the upper respiratory tract of swine and includes strains with different degree of virulence (Galofré-Milà et al., 2017). Basically, all piglets are colonized by the bacterium, but only certain types of strains are capable of causing disease, often following a perturbation to their host (e.g. co-infections, stress, medication) (Aragon et al., 2012).

Pathogenesis of $H$. parasuis is a multifactorial process, which is associated with virulence genes, serovars, biofilm production, etc. Although it is controversial whether serovar of $H$. parasuis is an indicator of virulence, serotyping is still an important feature of Glässer's disease diagnosis, as it is necessary for veterinarians and farmers to formulate a vaccination strategy. To date, a total of 15 serovars (serovars 1 through 15 ) of $H$. parasuis have been defined using a gel immuno-diffusion assay as well as molecular serotyping method (the latter cannot discriminate between serovars 5 and 12) (Howell et al., 2015). Serovars 5 and 4 of $H$. parasuis are widely considered disease-causing serovars and are the most common serovars of $H$. parasuis isolated from clinically sick pigs worldwide (Oliveira and Pijoan, 2004). On the other hand, serovars 3 and 7 of 
$H$. parasuis are generally recognized as non-virulent, although they can be isolated from pathological samples and some reports also indicate that isolates from those serovars can cause disease (Aragon et al., 2010; Zhang et al., 2012; Costa-Hurtado et al., 2013). The prevalence and distribution of serovars of $H$. parasuis isolated from clinical cases has been widely studied but not so from those isolated from healthy carrier pigs.

In China and many other countries, antimicrobial agents are still largely used and play an important role for the prevention and treatment of bacterial diseases. Tilmicosin and florfenicol are the drugs of choice for prevention and treatment of $H$. parasuis infections in China. Historically, $H$. parasuis isolates showed high susceptibility to frequently used drugs (El Garch et al., 2016; Zhou et al., 2010), but recently multiple publications indicate a decreased susceptibility of $H$. parasuis to several antimicrobial agents from a number of countries (Brogden et al., 2018; Dayao et al., 2014). In China, the last nationwide surveillance of antimicrobial susceptibility of $H$. parasuis was reported in 2010 (Zhou et al., 2010), which showed that Chinese H. parasuis isolates were susceptible to most of the antimicrobial agents. However, isolates from healthy piglets has been shown to carry plasmids with antimicrobial resistance genes, which may play an important role in the spreading of these resistances (Moleres et al., 2015).

In order to understand the current situation of $H$. parasuis from healthy pigs, here, we investigated the prevalence and characteristics of this organism from 6 provinces in China during 2016-2017. Our findings provide more information for better understanding of the serovars and antimicrobial resistance distribution in H. parasuis. 


\section{Farms, animals and collection of samples}

110 From September 2016 to October 2017, nasal swabs were collected from

111 healthy pigs on 13 pig farms and 2 slaughterhouses located in 6 provinces

112 (Table 1). Samples were collected from different growth stages of pigs: sows,

113 weaned pigs and finishers. Nasal swabs were placed in tryptic soy broth (TSB)

114 containing nicotinamide adenine dinucleotide (NAD) $(10 \mu \mathrm{g} / \mathrm{ml})$ and $5 \%(\mathrm{v} / \mathrm{v})$

115 fetal calf serum (FCS), and then transported to the laboratory in a Styrofoam

116 container with ice packs (within one day). A brief questionnaire was collected

117 from each farm. The contents of the questionnaire included the type of pig

118 production, herd size, breeds of pigs, age of piglets and history of antimicrobial 119 usage.

121 Table 1. Source of $244 H$. parasuis isolates from healthy pigs 2016-2017.

\begin{tabular}{ccccc} 
Province & \multicolumn{2}{c}{ Percentage of isolation (No. of nasal samples) } & Total \\
& Sow & Weaner & Finisher & \\
Beijing & $1.2 \%(86)$ & $17.3 \%(52)$ & $16.7 \%(60)$ & $10.1 \%(198)$ \\
Shandong & $4.5 \%(134)$ & $18.3 \%(153)$ & $8.8 \%(114)$ & $11.0 \%(401)$ \\
Henan & $2.7 \%(37)$ & $22.7 \%(97)$ & - & $17.2 \%(134)$ \\
Shanghai & - & $31.0 \%(29)$ & $8.2 \%(158)$ & $11.8 \%(187)$ \\
Sichuan & $1.5 \%(68)$ & $12.8 \%(226)$ & $2.2 \%(89)$ & $8.4 \%(383)$
\end{tabular}




\begin{tabular}{|c|c|c|c|c|}
\hline \multicolumn{5}{|c|}{ Percentage of isolation (No. of nasal samples) } \\
\hline \multirow{2}{*}{ Province } & & & & \multirow{2}{*}{ Total } \\
\hline & Sow & Weaner & Finisher & \\
\hline Chongqing & $0 \%(38)$ & $32.5 \%(292)$ & $19.0 \%(42)$ & $27.7 \%(372)$ \\
\hline Total & $2.5 \%$ (363) & $22.6 \%(849)$ & $9.3 \%(463)$ & $14.6 \%(1675)$ \\
\hline
\end{tabular}

\section{Bacterial isolation and identification}

125 Nasal samples were vortexed vigorously upon arrival at the laboratory. A loopful

126 of suspension from each sample was streaked onto tryptic soy agar (TSA)

127 plates containing NAD $(10 \mu \mathrm{g} / \mathrm{ml})$ and $5 \%(\mathrm{v} / \mathrm{v}) \mathrm{FCS}$, and then incubated at $37^{\circ} \mathrm{C}$

128 for $36 \mathrm{~h}$. One or two suspect colonies from the agar plate were subjected to 129 further identification by polymerase chain reaction (PCR) as reported previously 130 (Oliveira et al., 2001).

\section{DNA preparation and serotyping}

133 The DNA templates for PCR amplification were prepared by boiling. Briefly, $1 \mathrm{ml}$ 134 of an overnight culture was spinned down at $12,000 \times \mathrm{g}$ for $5 \mathrm{~min}$ and the bacterial pellet was resuspended in $100 \mu \mathrm{l}$ TE buffer $(10 \mathrm{mM}$ Tris- $\mathrm{HCl}, 1 \mathrm{mM}$ EDTA, pH 8.0). The suspension was heated at $100^{\circ} \mathrm{C}$ for $10 \mathrm{~min}$, followed by

137 cooling on ice for $10 \mathrm{~min}$. After centrifugation at $12,000 \times \mathrm{g}$ for $5 \mathrm{~min}$, the 138 supernatant was transferred to a nucleic acid-free tube and stored at $20^{\circ} \mathrm{C}$. described one-step multiplex PCR (Howell et al., 2015). 


\section{Antimicrobial susceptibility testing}

143 Currently, the recommended method for antimicrobial susceptibility testing of $H$.

144 parasuis is not available in the Clinical and Laboratory Standards Institute

145 (CLSI) guidelines. The minimum inhibitory concentrations (MICs) of $H$. parasuis

to a panel of antimicrobial agents were determined by the broth microdilution method (Clinical and Laboratory Standards Institute, 2013) using cationadjusted Mueller-Hinton broth containing NAD $(25 \mu \mathrm{g} / \mathrm{ml})$ and $1 \%(\mathrm{v} / \mathrm{v})$ sterile filtered heat-inactivated chicken serum as suggested by a previous report

150 (Prüller et al., 2017). Antimicrobial agents and concentrations tested were: amoxicillin/clavulanic acid (0.06/0.03-64/32 $\mu \mathrm{g} / \mathrm{ml})$, ceftiofur $(0.015-16 \mu \mathrm{g} / \mathrm{ml})$, enrofloxacin $(0.015-16 \mu \mathrm{g} / \mathrm{ml})$, florfenicol $(0.06-64 \mu \mathrm{g} / \mathrm{ml})$, erythromycin $(0.12-$ $128 \mu \mathrm{g} / \mathrm{ml})$, tilmicosin $(0.12-128 \mu \mathrm{g} / \mathrm{ml})$, doxycycline $(0.06-64 \mu \mathrm{g} / \mathrm{ml})$, and tetracycline $(0.12-128 \mu \mathrm{g} / \mathrm{ml})$. Escherichia coli ATCC 25922 and Staphylococcus aureus ATCC 29213 were used as quality control strains.

The $\mathrm{MIC}_{50}$ and $\mathrm{MIC}_{90}$ were defined as the MIC value at which $\geq 50 \%$ and $\geq 90 \%$ of the isolates within a test population are inhibited. If the resulting number was not an integer, the next integer following the respective value represented the MIC50 and MIC90 (Schwarz et al., 2010).

\section{Data analysis}

The comparison between categorical variables was carried out via $\mathrm{X} 2$ test using GraphPad prism v7.0. P values lower than 0.05 were considered significant. 


\section{Prevalence of $\boldsymbol{H}$. parasuis}

167 During the study period, a total of $244 H$. parasuis isolates were obtained from 1681675 nasal samples (Table 1): 9 isolates from sows, 192 isolates from weaners 169 and 43 isolates from finishers. The overall isolation rate was $14.6 \%(244 / 1675)$.

170 Highest prevalence was observed in weaners (22.6\%, 192/849), followed by 171 finishers $(9.3 \%, 43 / 463)$ and sows $(2.5 \%, 9 / 363)$.

172

\section{Serovar distribution}

174 Using the multiplex PCR method, 244 isolates were assigned to 12 serovars, 175 and 8 isolates were non-typeable. The highest prevalence was observed for serovar $7(20.1 \%, 49 / 244)$, followed by serovar $3(14.8 \%, 36 / 244)$, serovar 2

$177(14.3 \%, 35 / 244)$, serovar $11(12.7 \%, 31 / 244)$, serovars $5 / 12(5.7 \%, 14 / 244)$ and 178 serovar $4(2.5 \%, 6 / 244)$ (Fig. 1). Serovars 14 and 15 were not detected. 


\section{a}

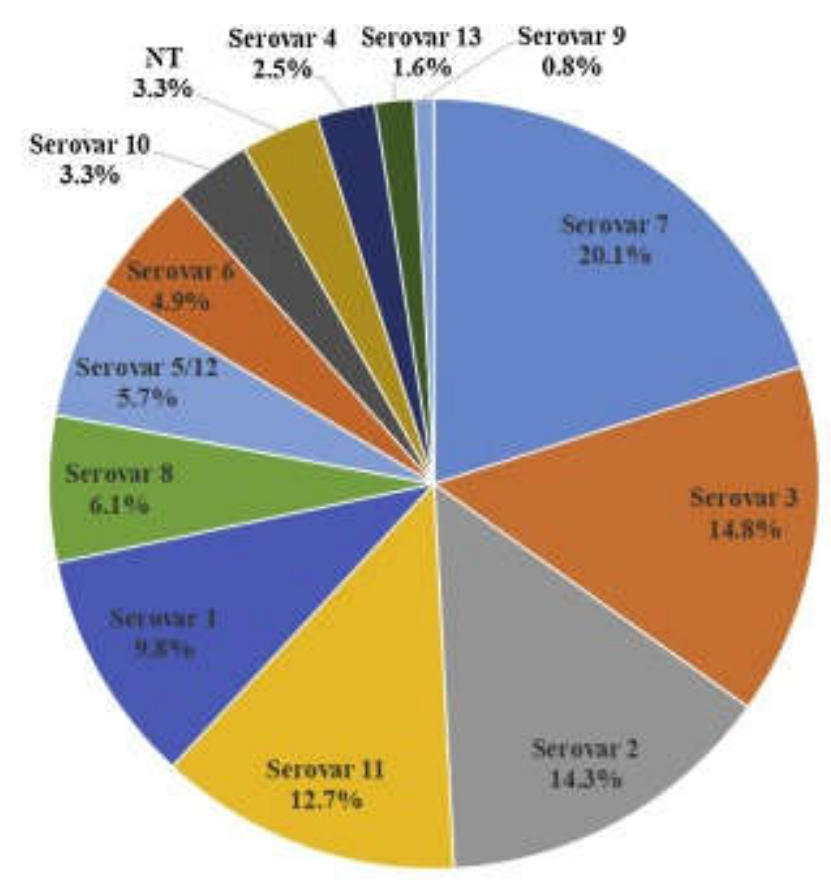

b

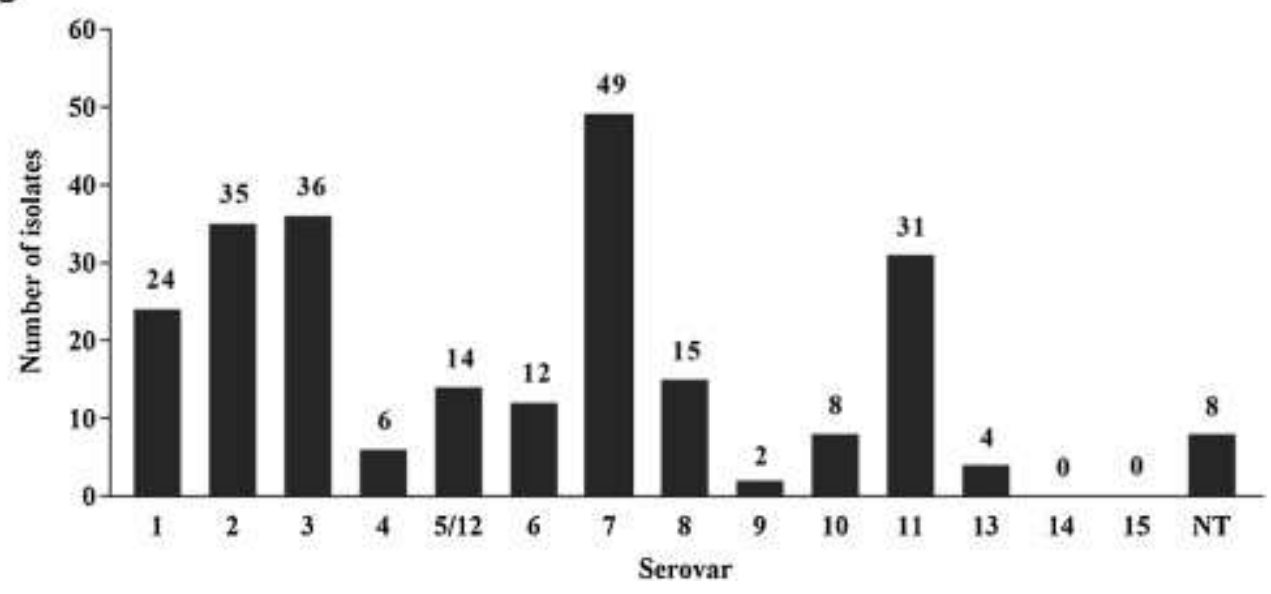

Fig. 1. Serovar distribution of $244 H$. parasuis isolates from healthy pigs by (a) percentages and (b) number of isolates. NT means non-typeable.

As shown in Fig. 2, serovars 1 and 3 were detected from samples from all the 6 provinces, while serovar 11 was detected only from Chongqing and Beijing. Most of the serovar 2 isolates were from Shandong, Shanghai and Beijing. For serovar 3, most of the isolates were from Sichuan and Chongqing. 


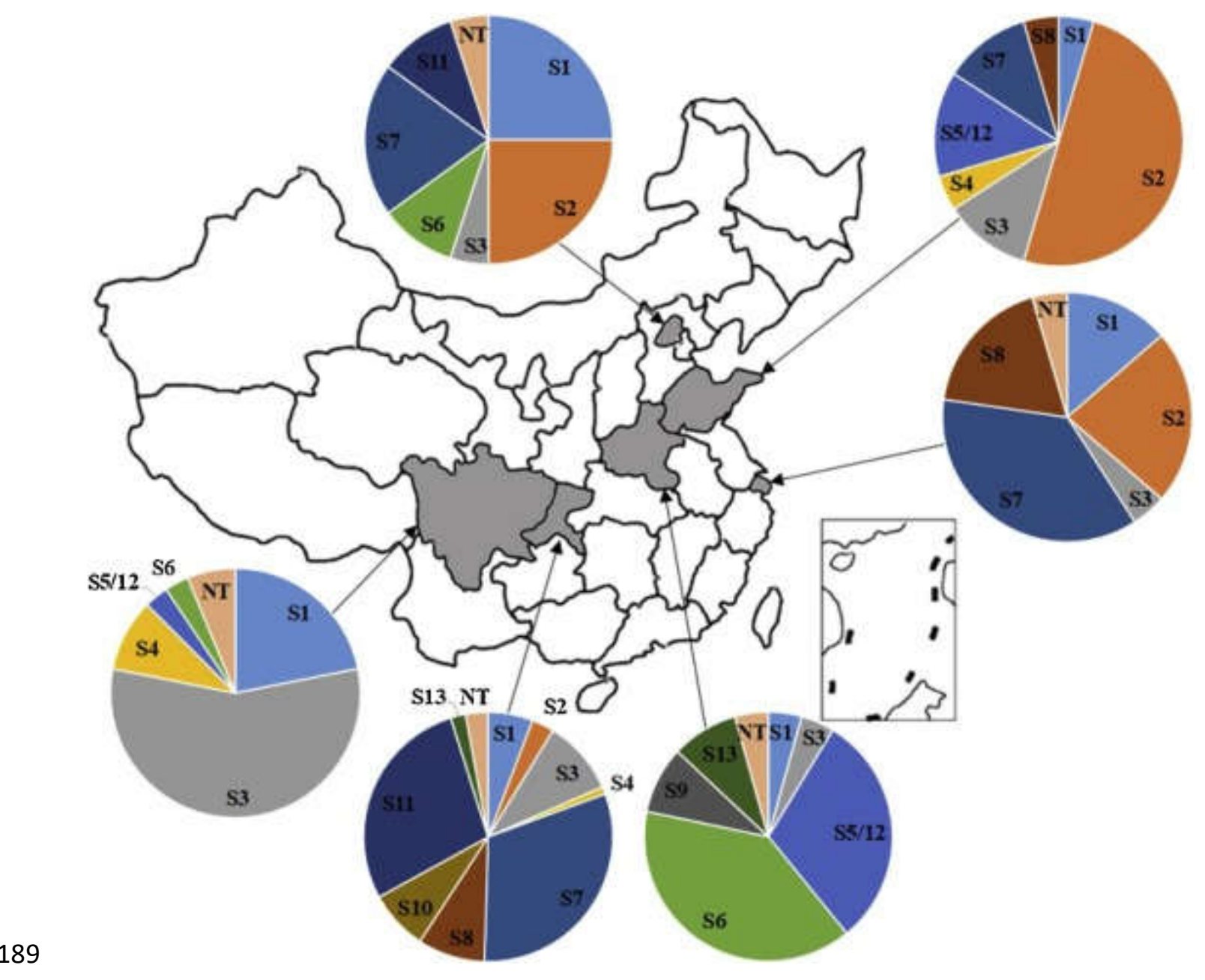

190 Fig. 2. Geographic distribution of serovars of 244 H. parasuis isolates from

191 healthy pigs in 6 provinces of China. The pie chart showed the proportion of the serovar type in each province. NT means non-typeable.

\section{Antimicrobial susceptibility profiles}

The MIC distribution of 8 antimicrobials for the $244 H$. parasuis isolates are

197 detected for enrofloxacin, florfenicol, erythromycin, tetracycline, tilmicosin, and 198 doxycycline. 
199 Table 2. MIC distribution of $244 \mathrm{H}$. parasuis isolates from healthy pigs 20162002017

Antimicrobial agent

No. of isolates with MIC values $(\mu \mathrm{g} / \mathrm{ml})$ of:

$$
0.0080 .0150 .030 .060 .120 .250 .51248163264128256 \text { MIC }_{50} \text { MIC }_{90}
$$

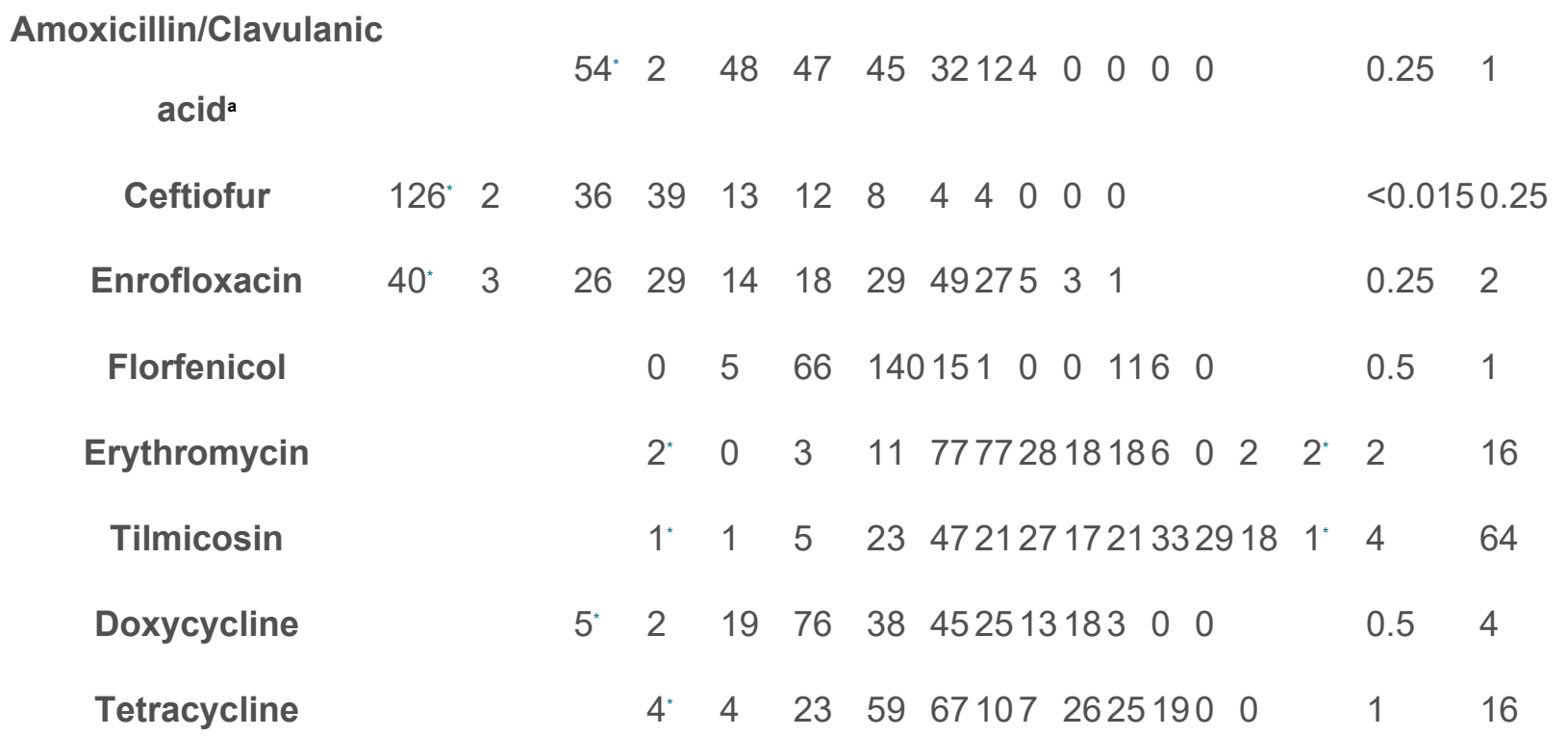

$201 \quad a$

202 Concentrations for amoxicillin given, tested with clavulanic acid in a concentration ratio 2:1.

203

204

Number of isolates with MIC values equal to or higher/lower than concentrations of the test

205

range. The white areas represent the tested range of an antimicrobial agent.

206

207 The $\mathrm{MIC}_{50}$ and $\mathrm{MIC}_{90}$ values for $H$. parasuis were also calculated (Table 2). For

208 antimicrobial agents most frequently used in preventing and treating diseases

209 caused by H. parasuis (e.g. tilmicosin and florfenicol), elevated MICs were

210 observed compared to that from previous reports (Zhou et al., 2010). For

211 antimicrobial agents less frequently used for the treatment of $H$. parasuis 
212 infections, the $\mathrm{MIC}_{90}$ and $\mathrm{MIC}_{50}$ values were relatively low. For example, $\mathrm{MIC}_{50}$ and $\mathrm{MIC}_{90}$ values of ceftiofur were $0.015 \mu \mathrm{g} / \mathrm{ml}$ and $0.25 \mu \mathrm{g} / \mathrm{ml}$, respectively.

\section{Discussion}

$H$. parasuis is one of the most important pathogens in pigs. Certain serovars of this organism are clearly associated with clinical infections. In this study, we obtained $244 \mathrm{H}$. parasuis isolates from 1675 nasal samples from healthy pigs across 6 provinces. We also characterized the serovars and antimicrobial susceptibility of the $H$. parasuis isolates. Our result indicated the high prevalence and increased antimicrobial resistance of $H$. parasuis from healthy pigs. In addition, pathogenic serovars were observed in these isolates.

223 The overall prevalence by culture (14.6\%) of $H$. parasuis among healthy pigs in this study was slightly lower than that found in diseased pigs $(22.1 \%)$ in a previous report (Cai et al., 2005), but this discordance can be explained by the differences between both studies. Cai et al. (2005) included isolates collected from lesions of diseased pigs, which more probably were weaned pigs, while in our study, samples were obtained from the nasal cavities of healthy sows, weaners and finishers. Our results confirm that the prevalence of $H$. parasuis in weaned pigs was significantly higher than in finisher pigs $(22.6 \%$ vs $9.3 \%, \mathrm{P}<$ $0.0001)$ and sows $(22.6 \%$ vs $2.5 \%, \mathrm{P}<0.0001)$, as previously reported (Angen et al., 2007; Cerdà-Cuéllar et al., 2010). Moreover, even in weaned pigs, the isolation rate varied between different farms, ranging from $0 \%$ to $51.6 \%$ (data not shown). The phenomenon was also observed in previous reports (Maclnnes et al., 2008; Turni and Blackall, 2010). 
Serovars 7, 3, 2, and 11 were the most prevalent serovars (all above 10\%), which are largely different from previous reports with clinical isolates, where serovars 5 and 4 were the predominant serovars (Angen et al., 2004; Cai et al., 2005; Castilla et al., 2012; Ma et al., 2016). The difference is probably due to the sampling sites: all the nasal samples in this study were collected from healthy pig herds, and in other studies the samples were lungs, fluids from joints, etc. which were obtained from sick pigs. Serovars 7,3 , and 11 were used to be considered as avirulent, and serovar 2 as moderate virulent according to challenge experiment using a small number of isolates (Aragon et al., 2010; Kielstein and Rapp-Gabrielson, 1992). In fact, serovars 3 and 11 isolates were also reported virulent or even highly virulent (Aragon et al., 2010; Dai et al., 2016). Serovars 5 and 4 isolates are widely considered disease-causing strains, and they were most commonly isolated from sick pigs with Glässer's disease (Howell et al., 2014; Tadjine et al., 2004). In the present study, serovars 5/12 and 4 were detected in a low percentage $(5.7 \%$ and $2.5 \%$ for $5 / 12$ and 4 , respectively). However, this low isolation of serovars $5 / 12$ and 4 in healthy herds can be potentially significant because these isolates may epidemically spread under certain circumstances. It was mentioned that 93.5\% (29/31) of serovar $11 \mathrm{H}$. parasuis were isolated from Chongqing province, which showed an obvious regional difference.

MICs distribution is one of the most important parameters for reporting results of antimicrobial susceptibility testing (Schwarz et al., 2010). If the MICs distribution is bimodal or multimodal, it usually indicates the presence of resistance mechanism/s (Morrissey et al., 2014). The MICs of most of the antibiotics in our study showed bimodal or multimodal distributions (enrofloxacin, florfenicol, 
erythromycin, tetracycline, tilmicosin, and doxycycline). For example, florfenicol

MICs showed a bimodal distribution, and isolates with MICs $>4 \mu \mathrm{g} / \mathrm{ml}$

(distributed around the second peak) were proved to harbor the phenicol macrolide resistance mechanisms cannot completely account for the multimodal distribution of tilmicosin MICs (data not published), which might imply a new tilmicosin resistance mechanism. This was of great concern, as nasal isolates were considered as an important reservoir for antimicrobial resistance. Bimodal or multimodal distributions of tilmicosin MICs were also reported in Germany and Australia recently (Brogden et al., 2018; Dayao et al., 2014, 2016).

For most of the tested antimicrobials, elevated MICs were observed compared to previous reports. For example, tilmicosin $\mathrm{MIC}_{90}$ values were $64 \mu \mathrm{g} / \mathrm{ml}$ in our study, which are 16-fold higher than that in Germany (Brogden et al., 2018) and 32-fold higher than previously described in China (Zhou et al., 2010). Florfenicol MIC $_{90}$ values $(1 \mu \mathrm{g} / \mathrm{ml})$ were similar to those in Germany $(0.5 \mu \mathrm{g} / \mathrm{ml})$ and China in $2010(1 \mu \mathrm{g} / \mathrm{ml})$. However, no isolates had MICs $>1 \mu \mathrm{g} / \mathrm{ml}$ in the two MICs $>8 \mu \mathrm{g} / \mathrm{ml}$.

\section{Conclusions}

In conclusion, serovar distribution was different from previous reports with

282 clinical isolates and showed regional differences. Elevated MICs of $H$. parasuis

283 for most of the antimicrobial agents were possibly due to the spread of multiple resistance mechanisms by the selection of antibiotics, especially for those 
285 frequently used for preventing and treating diseases caused by this genus, which highlighted the importance of using antimicrobials more prudently. All these findings provide us an overview of current epidemiological status of $H$. parasuis and can be helpful for the prevention and understanding of the disease caused by this bacterium.

290

291

\section{Funding}

292 The study was supported by grants from National Key Research and

293 Development Program of China (2016YFD0501304 and 2016YFD0501305).

\section{Acknowledgements}

We are grateful for the sampling support of Weiyong He in China Agricultural University and microbiologists in these agencies: Chongqing Academy of Animal Sciences, Sichuan Provincial Agricultural Department, Sichuan Agricultural University, Qingdao Agricultural University and Henan Agricultural University.

\section{References}

$\varnothing$. Angen, B. Svensmark, K.R. Mittal. Serological characterization of Danish

Haemophilus parasuis isolates.et. Microbiol., 103 (2004), pp. 255-258 improved species specific PCR test for detection of Haemophilus parasuis. Vet. 
309 (2016).

310 https://www.gov.uk/government/uploads/system/uploads/attachment_data/file/549113/

311 pub-survrep-p0216.pdf

312 V. Aragon, M. Cerdà-Cuéllar, L. Fraile, M. Mombarg, M. Nofrarías, A. Olvera, M. Sibila,

313 D. Solanes, J. Segalés. Correlation between clinico-pathological outcome and typing of 314 Haemophilus parasuis field strains. Vet. Microbiol., 142 (2010), pp. 387-393

315 V. Aragon, J. Segales, S. Oliveira. Glasser's disease. J. Zimmerm, L. Karrker, A.

316 Ramirez, K. Schwarz, G. Stevenson (Eds.), Diseases of Swine, John Wiley \& Sons, 317 Inc, lowa, USA (2012), pp. 760-770

318 S. Brogden, A. Pavlović, R. Tegeler, H. Kaspar, N. De Vaan, C. Kehrenberg.

319 Antimicrobial susceptibility of Haemophilus parasuis isolates from Germany by use of a 320 proposed standard method for harmonized testing. Vet. Microbiol., 217 (2018), pp. 3232135

322 X. Cai, H. Chen, P. Blackall, Z. Yin, L. Wang, Z. Liu, M. Jin. Serological

323 characterization of Haemophilus parasuis isolates from China. Vet. Microbiol., 111

324 (2005), pp. 231-236

K.S. Castilla, D.D.S. de Gobbi, L.Z. Moreno, R. Paixão, T.A. Coutinho, J.L. dos Santos,

A.M. Moreno. Characterization of Haemophilus parasuis isolated from Brazilian swine through serotyping, AFLP and PFGE. Res. Vet. Sci., 92 (2012), pp. 366-371

M. Cerdà-Cuéllar, J. Naranjo, A. Verge, M. Nofrarias, M. Cortey, A. Olvera, J. Segalés, V. Aragon. Sow vaccination modulates the colonization of piglets by Haemophilus parasuis. Vet. Microbiol., 145 (2010), pp. 315-320 
331 Clinical and Laboratory Standards Institute. Performance Standards for Antimicrobial

332 Disc and Dilution Susceptibility Test for Bacteria Isolated From Animals-Fourth Edition:

333 Approved Standard VET01-A4. CLSI, Wayne, PA, USA (2013)

334 M. Costa-Hurtado, A. Olvera, V. Martinez-Moliner, N. Galofré-Milà, P. Martínez, J.

335 Dominguez, V. Aragon. Changes in macrophage phenotype after infection of pigs with 336 Haemophilus parasuis strains of different virulence. Infect. Immun. (2013). IAI. 00056$337 \quad 00013$

K. Dai, J. Jin, Y. Wen, X. Wen, L. He, S. Cao, X. Huang, R. Wu, Q. Zhao. Complete genome sequence of highly virulent Haemophilus parasuis serotype 11 strain SC1401. Genome Announc., 4 (2016) e00628-00616

D.A.E. Dayao, M. Kienzle, J.S. Gibson, P.J. Blackall, C. Turni. Use of a proposed antimicrobial susceptibility testing method for Haemophilus parasuis. Vet. Microbiol., 172 (2014), pp. 586-589

D.A.E. Dayao, J.M. Seddon, J.S. Gibson, P.J. Blackall, C. Turni. Whole genome sequence analysis of pig respiratory bacterial pathogens with elevated minimum inhibitory concentrations for macrolides. Microb. Drug Resist., 22 (2016), pp. 531-537 Haag-Diergarten, A. Richard-Mazet, V. Thomas. Monitoring of antimicrobial susceptibility of respiratory tract pathogens isolated from diseased cattle and pigs across Europe, 2009-2012: VetPath results. Vet. Microbiol., 194 (2016), pp. 11-22 virulent strains of Haemophilus parasuis. BMC Vet. Res., 13 (2017), p. 124 US swine production systems. Swine news, 30 (2007), pp. 85-89 
K.J. Howell, L.A. Weinert, R.R. Chaudhuri, S.L. Luan, S.E. Peters, J. Corander, D.

Harris, $\varnothing$. Angen, V. Aragon, A. Bensaid et al. The use of genome wide association methods to investigate pathogenicity, population structure and serovar in Haemophilus parasuis. BMC Genomics, 15 (2014), p. 1179

K.J. Howell, S.E. Peters, J. Wang, J. Hernandez-Garcia, L.A. Weinert, S.L. Luan, R.R. Chaudhuri, $\varnothing$. Angen, V. Aragon, S.M. Williamson, et al. Development of a multiplex PCR assay for rapid molecular serotyping of Haemophilus parasuis. J. Clin. Microbiol., 53 (2015), pp. 3812-3821

P. Kielstein, V.J. Rapp-Gabrielson. Designation of 15 serovars of Haemophilus parasuis on the basis of immunodiffusion using heat-stable antigen extracts. J. Clin. Microbiol., 30 (1992), pp. 862-865

L. Ma, L. Wang, Y. Chu, X. Li, Y. Cui, S. Chen, J. Zhou, C. Li, Z. Lu, J. Liu. Characterization of Chinese Haemophilus parasuis isolates by traditional serotyping and molecular serotyping methods. PLoS One, 11 (2016), Article e0168903

J.I. Maclnnes, M. Gottschalk, A.G. Lone, D.S. Metcalf, S. Ojha, T. Rosendal, S.B. Watson, R.M. FriendshipPrevalence of Actinobacillus pleuropneumoniae, Actinobacillus suis, Haemophilus parasuis, Pasteurella multocida, and Streptococcus suis in representative Ontario swine herds. Can. J. Vet. Res., 72 (2008), p. 242

J. Moleres, A. Santos-López, I. Lázaro, J. Labairu, C. Prat, C. Ardanuy, B. GonzálezZorn, V. Aragon, J. Garmendia. Novel blaROB-1-bearing plasmid conferring resistance to $\beta$-lactams in Haemophilus parasuis isolates from healthy weaning pigs. Appl. Environ. Microbiol., 81 (2015), pp. 3255-3267

I. Morrissey, M.R. Oggioni, D. Knight, T. Curiao, T. Coque, A. Kalkanci, J.L. Martinez, B. Consortium. Evaluation of epidemiological cut-off values indicates that biocide resistant subpopulations are uncommon in natural isolates of clinically-relevant microorganisms. PLoS One, 9 (2014), Article e86669 
S. Oliveira, C. Pijoan. Haemophilus parasuis: new trends on diagnosis, epidemiology and control. Vet. Microbiol., 99 (2004), pp. 1-12

S. Oliveira, L. Galina, C. Pijoan. Development of a PCR test to diagnose Haemophilus parasuis infections. J. Vet. Diagn. Invest., 13 (2001), pp. 495-501

S. Prüller, C. Turni, P.J. Blackall, M. Beyerbach, G. Klein, L. Kreienbrock, K. method for broth microdilution susceptibility testing of Haemophilus parasuis. J. Clin. Microbiol., 55 (2017), pp. 264-273

S. Schwarz, P. Silley, S. Simjee, N. Woodford, E. van Duijkeren, A.P. Johnson, W. Gaastra. Assessing the antimicrobial susceptibility of bacteria obtained from animals. J. Antimicrob. Chemother., 65 (2010), pp. 601-604

M. Tadjine, K. Mittal, S. Bourdon, M. Gottschalk. Development of a new serological test for serotyping Haemophilus parasuis isolates and determination of their prevalence in North America. J. Clin. Microbiol., 42 (2004), pp. 839-840

C. Turni, P. Blackall. Serovar profiling of Haemophilus parasuis on Australian farms by sampling live pigs. Aust. Vet. J., 88 (2010), pp. 255-259 Prevalence and characterization of genotypic diversity of Haemophilus parasuis isolates from southern China. Can. J. Vet. Res., 76 (2012), pp. 224-229 antimicrobial resistance among different serovars of Haemophilus parasuis isolates. Vet. Microbiol., 141 (2010), pp. 168-173 